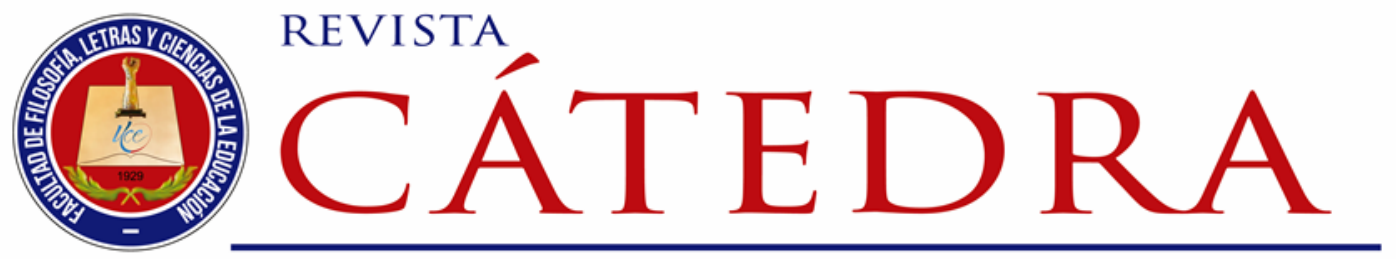

\title{
Análisis de la producción científica del Ecuador a través de la plataforma Web of Science
}

\section{Analysis of the scientific production in Ecuador through the web platform on science}

\author{
Elking Araujo-Bilmonte \\ Pontificia Universidad Católica del Ecuador, Quito, Ecuador \\ earaujo@puce.edu.ec \\ https://orcid.org/0000-0001-9206-7883 \\ Liceth Huertas-Tulcanaza \\ Universidad Central del Ecuador, Quito, Ecuador \\ lkhuertas@uce.edu.ec \\ https://orcid.org/0000-0001-6216-1238 \\ Kenny Párraga-Stead \\ Universidad Central del Ecuador, Quito, Ecuador \\ ksparraga@uce.edu.ec \\ https://orcid.org/0000-0002-8177-2066
}

(Recibido: 18/02/2020; Aceptado: 22/02/2020; Versión final recibida: 15/03/2020)

Cita del artículo: Araujo-Bilmonte, E., Huertas-Tulcanaza, L. y Párraga Stead, K. (2020). Análisis de la producción científica del Ecuador a través de la plataforma Web of Science, Revista Cátedra, 3(2), 150-165.

\section{Resumen}

El presente documento nace de la desventaja encontrada en la producción científica de Ecuador con respecto a otros países de Latinoamérica. El objetivo de esta investigación es reflexionar sobre la importancia de la producción científica para el desarrollo social, educativo y científico del Ecuador. La metodología usada en esta investigación es de carácter inductiva - deductiva y se apoya en la investigación bibliográfica utilizando el método hermenéutico. Se presentan los resultados obtenidos en producción científica de la

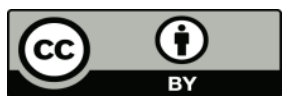


plataforma Web of Science de los períodos 2006 - 2008 y 2015 - 2020 en Latinoamérica. Se toman como referencia estos períodos para contrastar los registros de producción científica previos a la reforma universitaria en Ecuador en el 2008 con los actuales. De entre los resultados, se determina un incremento en la producción científica de Ecuador en el último período. Ecuador se encuentra en el puesto 7 de los 20 países analizados. Se concluye que Ecuador muestra un crecimiento en la producción científica al igual que los otros países. Sin embargo, aún existe una diferencia significativa con respecto a: Brasil, México, Argentina, Chile y Colombia. A partir del año 2008, se estableció un proceso de evaluación de las instituciones de educación superior que ha influido positivamente en la producción científica de Ecuador. Además, estas cifras se pueden mejorar cambiando el paradigma educativo para que la escritura científica sea tomada como un eje transversal desde los primeros años de educación formal.

Palabras clave

Difusión, Ecuador, investigación, Latinoamérica, publicación, Web of Science.

\section{Abstract}

This document arises from the disadvantage found in the scientific output of Ecuador in relation to other countries in Latin America. The purpose of this investigation is reflecting about the importance of the scientific output for the social, educational and scientific development of Ecuador. The methodology used in this investigation is inductive deductive and it is supported by bibliographical research using the hermeneutic method. The results obtained of the scientific output from the Web of Science platform during the periods 2006 - 2008 and 2015 - 2020 in Latin America are presented. These periods are taken as a reference to contrast the records of scientific production prior to the university reform in Ecuador in 2008 with the current ones. Among the results, it is observed an increase in the scientific output in the country during the last period. Ecuador is in the $7^{\text {th }}$ position among the 20 analyzed countries. It is concluded that Ecuador shows a growth in the scientific output as well as the other countries. However, there is still a significant difference with: Brazil, Mexico, Argentina, Chile and Colombia. Since 2008, an evaluation process of the Higher Education Institutions was established and it has positively influenced the scientific output of Ecuador. Furthermore, these numbers can be improved by changing the educational paradigm in which writing would be taken as a traversal axis since the first years of formal education.

\section{Keywords}

Dissemination, Ecuador, investigation, Latin America, publication, Web of Science.

\section{Introducción}

La actividad científica presenta un incremento significativo que ha generado la necesidad de plantear indicadores para su medición. El seguimiento permanente de estos indicadores es de interés para mejorar las políticas científicas de los países y contribuir con su desarrollo. La producción científica hace referencia al nuevo conocimiento generado y a su difusión. En este sentido, existen bases de datos, entre ellas Web of Science, que registran estos valores y que permiten analizar la situación de los países en cuanto a difusión de la investigación.

Latinoamérica es una región que presenta diferentes matices en cuanto a investigación. Álvarez-Muñoz y Pérez-Montoro (2016) señalan que "estos países están en un momento de desarrollo y adaptación a los esquemas de evaluación y calidad que existen en otros países

\section{Licencia Creative Commons Atribución 4.0 Internacional (CC BY 4.0)}


más avanzados en el campo de la ciencia" (p. 758). Lo antes mencionado está relacionado con las variaciones de los países latinoamericanos en cuanto a la producción académica. En los últimos años, algunos de estos países han obtenido una visibilidad importante a nivel internacional.

En Ecuador, a partir del año 2008, por medio del Mandato 14, las instituciones de educación superior (IES) entraron en un proceso de evaluación que tuvo como objetivo categorizar a las IES para garantizar la calidad de la educación. Se desarrolló la investigación tomando como indicador las publicaciones. El CEAACES1 en 2014 afirma: "Las publicaciones científicas de las y los docentes de la IES y que, en principio, se supone presentan los avances y alcances de sus actividades investigativas" (p.143). Razón por lo cual, el número de publicaciones científicas de los docentes constituye actualmente un factor que da la acreditación a las IES del país.

Con este antecedente, la producción académica ha ganado mayor importancia a nivel de la educación superior. Sin embargo, ha representado un gran problema por el escaso conocimiento de los docentes y estudiantes para realizar una producción escrita de carácter académico y científico. Además, "la evaluación del criterio estudiantes arroja una universidad ecuatoriana fragmentada por dos brechas: la democrática y la tecnológica" (CEAACES, 2014, p. 136). Lo mencionado hace referencia a la deficiencia del sistema administrativo universitario y del acceso a recursos tecnológicos. Siendo estos obstáculos para el desarrollo de investigaciones en las IES. Por esto se plantearon estrategias para subsanar estas falencias y mejorar la producción científica.

Una de las dificultades principales en este trabajo de investigación fue la escasez de información y seguimiento de la producción científica en la región. Además, Cañedo-Andalia et al., 2010 mencionan que:

En los países menos desarrollados, se puede apreciar una gran cantidad de artículos científicos que sólo se registran en bases de datos de cobertura nacional y, en algunos casos, en regionales, cuyos propósitos fundamentales son muy diferentes a los de las referidas bases de datos internacionales (p. 29).

Ecuador y otros países latinoamericanos están en crecimiento y se han insertado en la visión mundial, pero es importante que se realicen investigaciones de producción científica a nivel nacional o regional. Este documento presenta la producción científica de Ecuador con respecto a los otros países latinoamericanos según la Web of Science. Esta plataforma almacena información de importantes bases de datos de carácter internacional. Principalmente, España utiliza la Web of Science para medir su producción científica. También existe un número limitado de estudios que haya tomado como referencia los datos de la Web of Science. Sin embargo, se requiere un panorama más amplio en cuanto a trabajos relacionados para hacer una comparación y discusión de los datos extraídos.

${ }^{1}$ CEAACES: Consejo de Evaluación, Acreditación y Aseguramiento en la Calidad cambió de nombre a Consejo de Aseguramiento de la Calidad de la Educación Superior (CACES) mediante la Ley Orgánica Reformatoria a la Ley Orgánica de Educación Superior que entró en vigor el 2 de agosto de 2018.

Licencia Creative Commons Atribución 4.0 Internacional (CC BY 4.0)

Revista Cátedra, 3(2), pp. 150-165, mayo-agosto 2020. e-ISSN: 2631-2875

https://doi.org/10.29166/10.29166/catedra.v3i2.2160 
El propósito de este documento es reflexionar sobre la importancia de la producción científica para el desarrollo educativo y científico del país utilizando los datos obtenidos de la plataforma Web of Science. Con este fin, se busca las respuestas a las siguientes preguntas:

- ¿Cuál es la situación de Ecuador con respecto a los otros países latinoamericanos en producción científica según la Web of Science?

- ¿Qué cambios se han registrado en Ecuador en producción científica a partir del Mandato 14 ?

En cuanto a la estructura y contenido del documento, en la sección 2 se presentan los conceptos relacionados con la temática. En la sección 3, se exponen resultados de investigaciones o análisis anteriores. En la sección 4, se detalla la metodología utilizada para desarrollar este documento investigativo. En la sección 5, se muestran los resultados de los registros obtenidos en la plataforma Web of Science. En la sección 6, se presenta la discusión de resultados a través de un análisis de los datos obtenidos. Finalmente, en la sección 7 , se presentan las conclusiones de acuerdo con los resultados.

\section{Conceptos relacionados}

\subsection{Horizontes epistemológicos de la producción científica}

Se considera a la producción científica como la parte palpable de los conocimientos o ideas científicas, dando lugar a un proceso de construcción material del conocimiento. "La producción científica (PC) es considerada como la parte materializada del conocimiento generado, es más que un conjunto de documentos almacenados en una institución de información" (Piedra-Salomón y Martínez-Rodríguez, 2007, p. 33). Dicho de otro modo, la producción científica no es solo un registro documental de los conocimientos. Se puede considerar una disciplina que permite la estructuración de los saberes y hace posible la divulgación de estos. Aunque una de sus funciones es el registro documental del conocimiento, la definición de producción científica es mucho más extensa.

Los procesos de producción científica, pese a no ser recientes a lo largo de la historia, han tenido un incremento y mejora en los últimos años. Se puede decir que la producción científica está inmersa en los acontecimientos cotidianos de la humanidad.

Este fenómeno se encuentra ligado a la mayoría de los acontecimientos en los que se ven involucradas las personas, cotidianamente, por lo que la evaluación de la misma [sic], atendiendo al resultado de los trabajos de investigación e innovación, no es una práctica reciente en las diversas áreas disciplinares (Piedra-Salomón y Martínez-Rodríguez, 2007, p. 33).

En función del incremento de la producción científica, es necesario encontrar nuevas formas o herramientas que permitan que la información científica llegue a poseer una cobertura extensa. "El desenvolvimiento rápido y vertiginoso de la ciencia y la técnica ha generado un aumento sin precedentes de la literatura creando la necesidad de buscar nuevos caminos para la divulgación de la información científica producida" (Piedra-Salomón y MartínezRodríguez, 2007, p. 36). La velocidad con la que se desarrolla el conocimiento científico genera la necesidad de optar por distintos medios de divulgación en donde la escritura se vuelve una de las herramientas para divulgar los saberes mediante revistas y artículos, así como páginas web y foros.

Recordando que la producción científica debe ser divulgada, los medios por los cuales se transmite la información pueden ser de carácter formal e informal. "La divulgación de la PC puede efectuarse por canales formales e informales de comunicación pues lo que se busca

Licencia Creative Commons Atribución 4.0 Internacional (CC BY 4.0)

Revista Cátedra, 3(2), pp. 150-165, mayo-agosto 2020. e-ISSN: 2631-2875

https://doi.org/10.29166/10.29166/catedra.v3i2.2160 
es transmitir información [...]" (Piedra-Salomón y Martínez-Rodríguez, 2007, p. 36). Basándose en lo anterior, la forma en la que se comparta el conocimiento científico y el medio que se ocupe, por consiguiente, pasa a segundo plano debido a que lo importante es la transmisión del conocimiento.

Los índices de producción científica a nivel mundial siempre han estado liderados por las grandes potencias. La producción de artículos científicos, desde el 2008, ha experimentado un notable incremento en cuanto a los índices de citas constantes en la plataforma Web of Science. Según la UNESCO (2015) "entre 2008 y 2014, el número de artículos científicos incluidos en el índice de citas científicas de la plataforma 'Web of Science' de Thomson Reuters aumentó en un 23\%, pasando de 1.029 .471 a 1.270 .425 ” (p. 3). Por consiguiente, el número de artículos registrados en dicha plataforma se incrementó en 240.954 en un período aproximado de 6 años.

En Latinoamérica, destacan ciertos países en producción científica debido a la intervención de organizaciones que la promovieron.

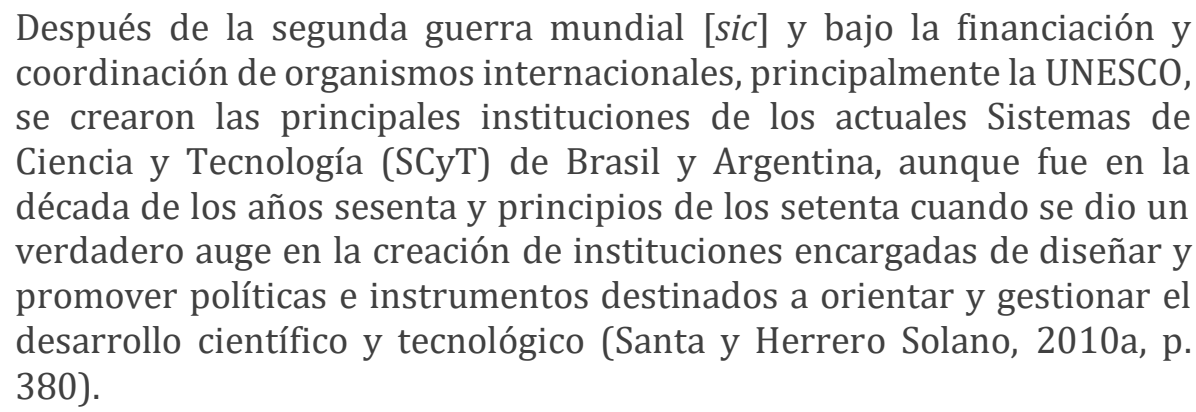

En este caso se observa que países como Argentina y Brasil poseen un contacto, desde el punto de vista histórico, con la producción científica. Los conflictos sociales suscitados en el siglo pasado permitieron la promoción del desarrollo científico incluso en países latinoamericanos. Por otro lado, el impulso de organizaciones internacionales como la UNESCO, como se evidencia en la cita anterior, fue un pilar fundamental para la producción científica en estos países.

Debido a la gran cantidad de factores problemáticos que existen en Latinoamérica, la producción científica se ve reducida considerablemente en la mayoría de estos países. Estos problemas se repiten en cada país de Latinoamérica complicando la producción de artículos académicos.

Con respecto a la producción académica de Latinoamérica, Santa y Herrero Solano (2010b) manifiestan que "es una de las regiones con mayor desequilibrio social y económico, situación que se traslada al campo de la producción científico-técnica" (p. 386). En otras palabras, los conflictos sociales, políticos y económicos afectan directamente en la producción científica provocando que la producción en la región sea dispareja. Además, estos problemas generan barreras que no permiten aumentar el índice de producción científica. Debido a estos factores la publicación de artículos académicos en Latinoamérica se ve reducida en ciertos períodos de tiempo.

Pese a todos estos problemas antes mencionados, en los últimos años América Latina y el Caribe han experimentado un incremento en la publicación de artículos académicos y producción científica como tal.

Licencia Creative Commons Atribución 4.0 Internacional (CC BY 4.0)

Revista Cátedra, 3(2), pp. 150-165, mayo-agosto 2020. e-ISSN: 2631-2875

https://doi.org/10.29166/10.29166/catedra.v3i2.2160 
Sin embargo, es reseñable el aumento que ha experimentado la región en el número de artículos publicados en los últimos años [...] en América Latina y el Caribe se publicaron 22.138 artículos en Scopus en el año 1996, mientras que está cifra aumentó a 126.620 en el año 2015, lo que supone un aumento de 5,72 puntos, mucho más que por ejemplo el aumento experimentado en Estados Unidos $(1,82)$ o Canadá $(2,31)$ (Guerrero-Casado, 2017, p. 28).

Es decir, que el índice de publicaciones, en cuanto a cantidad y en comparación con países norteamericanos ha aumentado considerablemente en un período de 20 años. Todos estos datos evidencian el incremento en cuanto a publicaciones académicas en Latinoamérica.

\subsection{Producción científica del Ecuador respecto a las normativas de control de las instituciones de educación superior}

El Estado ecuatoriano se considera un país de economía emergente debido a que la estabilidad económica del Ecuador está sostenida en su mayoría por la explotación y producción petrolera. "Ecuador es un país de economía pequeña [...] que está realizando esfuerzos considerables para ubicarse en el mapa de países creadores de conocimiento de calidad" (Álvarez-Muñoz y Pérez-Montoro, 2015, p. 578). El nivel económico y el desarrollo del país es un factor importante que influye en la producción y divulgación de textos académicos y conocimientos científicos. Pese a las dificultades económicas y sociales que se experimentan en el país y que se repiten en los demás países de América Latina, es importante mantener una extensa producción científica que funcione como medida para solucionar dichos conflictos.

Los siguientes trece años tras la finalización del siglo XX, Ecuador ha experimentado un ligero incremento en la producción científica, subiendo de posición en relación con los países de Latinoamérica. Álvarez-Muñoz y Pérez-Montoro (2015) en el análisis que realizaron en el 2014 señalan que "Ecuador se encuentra en la posición 12 en América Latina. En el período de análisis el país ha pasado de aportar al total latinoamericano el 0,39\% de producción científica en 2000 al 0,63\% en 2013" (p. 578). Tomando en cuenta lo mencionado, el país ha experimentado un incremento en su producción científica y académica.

Este incremento en producción científica se ve influenciado con los acontecimientos suscitados en el 2008 en relación con la reforma constitucional que vivió el país. A partir de esta reforma se genera una especial atención a los quehaceres universitarios con la sociedad.

El tratamiento limitado que dio el $\operatorname{CONESUP}^{2}$ a la solución de los problemas asociados con el desarrollo institucional y académico de las IES, junto con el poco interés demostrado por una gran mayoría de las IES en el cumplimiento de su responsabilidad social, al parecer fueron los detonantes para un cuestionamiento del quehacer universitario por parte de varios sectores sociales que justificaron de alguna manera la intervención del gobierno y de la Asamblea Nacional cuando trataron la Constitución de la República y la LOES, poniendo en evidencia que para

2 CONESUP: Consejo Nacional De Educación Superior cambió de nombre a Consejo de Educación Superior (CES) según la Ley Orgánica de Educación Superior del 12 de octubre de 2010.

Licencia Creative Commons Atribución 4.0 Internacional (CC BY 4.0)

Revista Cátedra, 3(2), pp. 150-165, mayo-agosto 2020. e-ISSN: 2631-2875

https://doi.org/10.29166/10.29166/catedra.v3i2.2160 
el caso ecuatoriano la Reforma Universitaria no nace de su seno sino, más bien, del reclamo social y gubernamental (Rojas, 2011, p. 60).

Con respecto a lo mencionado, se evidencian las causas por las cuales se presta atención a las actividades académicas de las universidades nacionales. De igual manera, es posible rescatar que la idea de la reforma universitaria se origina para satisfacer las necesidades de la sociedad y aportar al crecimiento de la misma.

De acuerdo con estos acontecimientos es posible afirmar que el Estado ecuatoriano presta vital atención a las actividades universitarias con la finalidad de generar una vinculación entre la realidad académica y la sociedad.

El Ecuador se encuentra en el proceso de acreditar a las universidades para mejorar los procesos de docencia, investigación y extensión. La necesidad de dar respuestas dinámicas al entorno, exige que se trabaje en el diseño de políticas participativas que coadyuven a que el vínculo universidad-sociedad transite hacia grados superiores (Cedeño, 2014, p. 22).

Es decir, el proceso de acreditación a las universidades ha permitido el incremento en cuanto a producción académica en el país. Adicionalmente, se habla de una mejora en los procesos docentes. La educación, en general, se inmiscuye en el proceso de desarrollo académico del país relacionado con la producción de trabajos investigativos y escritura académica.

Otro de los factores que ha permitido este impulso de la producción científica del Ecuador ha sido, como se mencionó anteriormente, las reformas constitucionales del 2008. La Constitución de la República del Ecuador en el artículo 351 expresa lo siguiente:

El sistema de educación superior estará articulado al sistema nacional de educación y al Plan Nacional de Desarrollo; [...] Este sistema se regirá por los principios de autonomía responsable, cogobierno, igualdad de oportunidades, calidad, pertinencia, integralidad, autodeterminación para la producción del pensamiento y conocimiento, en el marco del diálogo de saberes, pensamiento universal y producción científica tecnológica global (p. 60).

De esta manera, la Reforma Constitucional del 2008 incorpora a la producción científica dentro de las obligaciones de las instituciones educativas superiores. Además, se enlaza la realidad educativa universitaria con todo el sistema educativo del país exponiendo la necesidad de que los procesos académicos universitarios se vinculen con la sociedad en general. Estos hechos dan como resultado, desde el año 2008, un incremento en la producción de conocimientos científicos y escritura académica.

Dentro de la temática de políticas públicas inmersas en los procesos académicos, la Ley Orgánica de Educación Superior (LOES) juega un rol importante en el índice de producción científica del país. "En 2010, se promulgó la Ley Orgánica de Educación Superior (LOES) para promover la investigación en universidades" (Castillo y Powell, 2019, p. 11). Debido a esto, se evidencia un claro interés por la investigación y producción científica.

Licencia Creative Commons Atribución 4.0 Internacional (CC BY 4.0)

Revista Cátedra, 3(2), pp. 150-165, mayo-agosto 2020. e-ISSN: 2631-2875

https://doi.org/10.29166/10.29166/catedra.v3i2.2160 
En la LOES también se evidencia un claro interés por la labor docente y los niveles y porcentajes de publicaciones que deben tener en cuanto al ámbito académico. "La LOES insiste en que todos los profesores de la universidad deben tener al menos una maestría y el 70\% de ellos debe tener un doctorado en universidades de investigación" (Castillo y Powell, 2019, p. 11). En este caso, se plantea un requisito para formar parte del cuerpo docente en el nivel universitario. En consecuencia, los docentes que no poseen un doctorado tienen la necesidad de acceder a uno y por ende generar conocimientos científicos.

De igual manera, la Secretaría de Educación Superior, Ciencia, Tecnología e Innovación (SENESCYT) también se considera un organismo medular en la temática de producción científica a nivel de educación superior. En cierta medida, gracias a las políticas estipuladas por este organismo del Estado ha incrementado el índice de publicaciones académicas.

Durante los últimos años, la SENESCYT comenzó una política de acreditación de investigadores basada en productos. El incentivo alienta a los profesores a actualizar sus credenciales y aumentar las tasas de publicación para mejorar sus posibilidades de empleo y elevar su salario (Castillo y Powell, 2019, p. 11).

Por tanto, se puede entender el aumento de la producción científica en el Ecuador. Este incremento en las publicaciones de artículos académicos está relacionado con el impulso que brinda la SENESCYT a los docentes con la finalidad de mejorar su vida laboral y económica. De esta manera, se genera en los pedagogos la necesidad de producir conocimiento científico y publicarlo. En un principio, con la intención de mejorar el estilo de vida, pero que determina al mismo tiempo el crecimiento de los porcentajes de producción académica a nivel nacional.

\subsection{Plataforma Web of Science}

La Web of Science (WOS) es una plataforma web de la empresa Clarivate Analytics que recoge las referencias bibliográficas y citas de publicaciones científicas de cualquier disciplina del conocimiento. "Anteriormente era conocida como ISI Web of Knolewledge y era propiedad del Institute of Scientific Information. Después fue adquirida por Thomson Reuters" (UAM Biblioteca, 2019, pestaña Inicio). Por lo tanto, facilita el acceso a las bases de datos sobre las citas de documentos académicos. Su objetivo es proporcionar herramientas de análisis para valorar la calidad científica. En la plataforma se puede acceder a diferentes bases de datos a través de una interfaz única, de forma individual o a varias bases simultáneamente.

La WOS está compuesta por la colección básica Core Collection que abarca los índices de Ciencias, Ciencias Sociales y Artes y Humanidades, además de los Proceedings tanto de Ciencias como de Ciencias Sociales y Humanidades junto con las herramientas para análisis y evaluación, como son el Journal Citation Report y Essential Science Indicators. Adicionalmente, cuenta con las bases de datos que la complementan incluidas en la licencia para España: Medline, Scielo y Korean Citation Index (FECYT, 2019, sección Bases de Datos Web of Science).

La plataforma tiene acceso a diferentes bases de datos con la finalidad de contrastar información. Por tanto, podrá presentar un número de registros más neutro y evitar la repetición de artículos que aparecen en diferentes bases de datos. También contiene datos de los registros en todas las áreas del conocimiento. Así, es posible encontrar información con respecto a diversos temas.

Licencia Creative Commons Atribución 4.0 Internacional (CC BY 4.0)

Revista Cátedra, 3(2), pp. 150-165, mayo-agosto 2020. e-ISSN: 2631-2875

https://doi.org/10.29166/10.29166/catedra.v3i2.2160 
La información recogida por la plataforma es almacenada en colecciones de datos. "La Colección principal de Web of Science está formada por diez índices que incluyen información recopilada de miles de revistas académicas, libros, colecciones, informes y conferencias, entre otras muchas fuentes" (Clarivate, 2019, sección Ayuda de Web of Science). Estos índices son: Science Citation Index Expanded (SCI-EXPANDED), Social Sciences Citation Index (SSCI), Arts \& Humanities Citation Index (AH\&HCI), Conference Proceedings Citation Index - Science (CPCI-S), Conference Proceedings Citation Index Social Science \& Humanities (CPCI-SSH), Book Citation Index - Science (BKCI-S), Book Citation Index - Social Sciences \& Humanities (BKCI-SSH), Emerging Sources Citation Index (ESCI), Current Chemical Reactions (CCR-EXPANDED) e Index Chemicus (IC). Siendo así, una plataforma que contiene información de calidad sobre el proceso de investigación del mundo. Estos índices ayudan a la mejora de la producción científica y por ende, al desarrollo de la humanidad. Además, los números de registros muestran diferentes tipos de publicación o difusión de las investigaciones, generando cifras que representan de forma más precisa el nivel de investigación y desarrollo de ciencia en los países o regiones.

\section{Trabajos relacionados}

En el año 2007 se realizó una investigación que tenía como propósito definir el índice de publicaciones y producción científica en América Latina en los períodos de 1975 a 2004 en la Web of Science, En los resultados se determina un aumento del número total de trabajos en la región, siendo la colaboración Brasil-Argentina quien domina las colaboraciones bilaterales intra-regionales. Los autores llegan a la conclusión de que los índices de producción científica a nivel Latinoamericano se encuentran en constante ascenso y cobra impulso debido a las tendencias que existen a nivel mundial (Russell et. al, 2007).

En el año 2010 se realizó una investigación con el propósito de analizar los índices de publicación y visibilidad en la región de Latinoamérica y el Caribe en cuanto a bases de datos de la WOS en comparación con Scopus. Los resultados muestran que los países más pequeños no parecen crecer porque la cobertura de ciencia nacional sigue siendo muy limitada. Los autores llegaron a la conclusión de que existe un aumento en la producción y publicaciones de artículos científicos en la base de datos de revistas indexadas de SCImago Journal Rank (SJR) en contraste con los datos de Journal and Country Rank (JCR), pero este aumento no es significativo debido a lo reciente de las publicaciones (Santa y Herrero Solana, 2010a).

\section{Metodología}

A continuación, se describen los pasos utilizados en esta investigación:

1. Selección de los países para realizar el análisis comparativo: Se seleccionaron los 20 países latinoamericanos: Argentina, Bolivia, Brasil, Chile, Colombia, Costa Rica, Cuba, Ecuador, El Salvador, Guatemala, Honduras, México, Nicaragua, Panamá, Paraguay, Perú, Puerto Rico, República Dominicana, Uruguay y Venezuela.

2. Selección de los indicadores de producción científica: Los datos fueron obtenidos de la plataforma Web of Science y se tomó en cuenta los períodos de años: 2006 - 2008 y 2015 - 2020, debido al contraste existente de producción científica en los años anteriores a la reforma universitaria en el 2008 con la con la actualidad, y las bases de datos: Colección principal de Web of Science, Current

Licencia Creative Commons Atribución 4.0 Internacional (CC BY 4.0)

Revista Cátedra, 3(2), pp. 150-165, mayo-agosto 2020. e-ISSN: 2631-2875

https://doi.org/10.29166/10.29166/catedra.v3i2.2160 
Contents Connect, MEDLINE, ScieELO Citation Index, Korean Journal Database (KCI) y Russian Science Citation Index.

3. Análisis comparativo de los indicadores de producción científica durante el período 2006 - 2008: El análisis permite determinar las diferencias entre la producción científica, se ordenó de forma descendente a los países latinoamericanos según la producción académica de acuerdo al número de registros obtenidos desde la plataforma Web of Science.

4. Análisis comparativo de los indicadores de producción científica durante el período 2015 - 2020: El análisis permite determinar las diferencias entre la producción científica, se ordenó de forma descendente a los países latinoamericanos según la producción académica de acuerdo al número de registros obtenidos desde la plataforma Web of Science.

5. Análisis comparativo de la producción científica en las bases de datos durante el período 2015 - 2020: Se analizó la posición de Ecuador en producción científica con respecto a los otros países latinoamericanos en cada base de datos de la plataforma Web of Science.

6. Análisis comparativo de los indicadores de producción científica del período 2006 - 2008 y del período 2015 - 2020: Se compara los registros obtenidos durante los dos períodos en Web of Science para identificar las diferencias de la producción académica del Ecuador a partir de la reforma constituyente emitida en el 2008.

\section{Resultados}

La plataforma Web of Science muestra la producción científica de los países tomando en cuenta el número de registros. La información presentada fue recuperada el 8 de enero de 2020. En el Cuadro 1, se presenta la producción científica de los países latinoamericanos durante el período 2006 - 2008. Se presentan los países ordenados de forma descendente dependiendo del total de registros durante este tiempo. Se destaca con fondo gris los datos correspondientes a Ecuador.

\begin{tabular}{lrrrr}
\hline \multicolumn{1}{c}{ Países } & \multicolumn{4}{c}{ Producción científica durante el período } \\
\cline { 2 - 5 } & $\mathbf{2 0 0 6}$ & $\mathbf{2 0 0 7}$ & $\mathbf{2 0 0 8}$ & Total \\
Brasil & 37773 & 44169 & 50352 & 132294 \\
México & 16922 & 18643 & 20417 & 55982 \\
Argentina & 9383 & 10117 & 11608 & 31108 \\
Chile & 5861 & 6414 & 7264 & 19539 \\
Colombia & 4218 & 5148 & 6631 & 15997 \\
Venezuela & 2961 & 3134 & 3643 & 9738 \\
Cuba & 2161 & 2442 & 2685 & 7288 \\
Perú & 1178 & 1282 & 1413 & 3873 \\
Puerto Rico & 1145 & 1151 & 1398 & 3694 \\
Uruguay & 765 & 859 & 997 & 2621 \\
Costa Rica & 701 & 737 & 842 & 2280 \\
Ecuador & 430 & 479 & 560 & 1469 \\
Panamá & 392 & 466 & 500 & 1358 \\
Bolivia & 290 & 320 & 390 & 1000 \\
Paraguay & 183 & 178 & 189 & 550 \\
Guatemala & 115 & 135 & 146 & 396 \\
Nicaragua & 103 & 75 & 104 & 282 \\
\hline
\end{tabular}

Licencia Creative Commons Atribución 4.0 Internacional (CC BY 4.0) 


\begin{tabular}{lrrrr}
\hline El Salvador & 57 & 41 & 53 & 151 \\
Honduras & 49 & 38 & 56 & 143 \\
República & 6 & 9 & 8 & 23 \\
Dominicana & & & & \\
\hline
\end{tabular}

Cuadro 1. Producción científica de los países de Latinoamérica por años durante el período 2006 - 2008. Fuente: (Clarivate, 2020, Sección Búsqueda avanzada).

Según este número de registrosde la plataforma Web of Science se observa que Brasil es el país latinoamericano con más registros en total durante el período 2006 - 2008 (132294). A éste, le sigue México, Argentina, Chile, Colombia, Venezuela, Cuba, Perú, Puerto Rico, Uruguay y Costa Rica. A continuación, se encuentra Ecuador en el puesto 12 de la lista presentando un 1,11\% del número total de registros de Brasil. Finalmente, siguen en orden descendente: Panamá, Bolivia, Paraguay, Guatemala, Nicaragua, El Salvador, Honduras y República Dominicana.

En el Cuadro 2, se puede ver la lista de países ordenados de forma descendente en cuanto a producción científica a partir del número de registros obtenidos en la plataforma Web of Science del período 2015 - 2020.

\begin{tabular}{lrrrrrrr}
\hline \multicolumn{1}{c}{ Países } & \multicolumn{7}{c}{ Producción científica durante el período } \\
\cline { 2 - 8 } & $\mathbf{2 0 1 5}$ & $\mathbf{2 0 1 6}$ & $\mathbf{2 0 1 7}$ & $\mathbf{2 0 1 8}$ & $\mathbf{2 0 1 9}$ & $\mathbf{2 0 2 0}$ & \multicolumn{1}{c}{ Total } \\
Brasil & 81895 & 85656 & 91621 & 93510 & 89551 & 2564 & 444797 \\
México & 31505 & 33532 & 35248 & 35260 & 32670 & 925 & 169140 \\
Argentina & 17219 & 17612 & 18217 & 18654 & 16484 & 486 & 88672 \\
Chile & 14321 & 15724 & 16284 & 17135 & 15886 & 358 & 79708 \\
Colombia & 14104 & 15205 & 17250 & 16112 & 13698 & 387 & 76756 \\
Cuba & 4468 & 4554 & 4652 & 4583 & 2927 & 61 & 21245 \\
Ecuador & 2428 & 3594 & 5088 & 5248 & 4332 & 126 & 20816 \\
Perú & 3301 & 3507 & 4243 & 4401 & 3946 & 89 & 19487 \\
Uruguay & 1965 & 2188 & 2390 & 2455 & 2253 & 58 & 11309 \\
Venezuela & 2501 & 2464 & 2436 & 2042 & 1555 & 31 & 11029 \\
Costa Rica & 1615 & 1914 & 1969 & 1917 & 1644 & 41 & 9100 \\
Puerto Rico & 1392 & 1791 & 1656 & 1514 & 1377 & 43 & 7773 \\
Panamá & 867 & 905 & 973 & 941 & 988 & 18 & 4692 \\
Paraguay & 409 & 526 & 637 & 605 & 623 & 18 & 2818 \\
Bolivia & 627 & 605 & 527 & 499 & 507 & 24 & 2789 \\
Guatemala & 373 & 364 & 454 & 382 & 391 & 3 & 1967 \\
Nicaragua & 171 & 177 & 240 & 212 & 169 & 6 & 975 \\
Honduras & 144 & 122 & 183 & 208 & 227 & 11 & 895 \\
El Salvador & 174 & 151 & 192 & 184 & 151 & 4 & 856 \\
República & 97 & 99 & 97 & 124 & 179 & 4 & 600 \\
Dominicana & & & & & & & \\
\hline
\end{tabular}

Cuadro 2. Producción científica de los países de Latinoamérica durante el período 2015 - 2020. Fuente: (Clarivate, 2020, Sección Búsqueda avanzada). 
De acuerdo con este número de registros se observa que Brasil es el país latinoamericano con más registros en total durante el período 2015 - 2020 (444797) en la plataforma Web of Science. A éste, le sigue México, Argentina, Chile, Colombia y Cuba. A continuación, se encuentra Ecuador, en el puesto 7 de la lista presentando un 4,68 \% del número total de registros de Brasil. Finalmente, siguen en orden descendente: Perú, Uruguay, Venezuela, Costa Rica, Puerto Rico, Panamá, Paraguay, Bolivia, Guatemala, Nicaragua, Honduras, El Salvador y República Dominicana.

A continuación, en el Cuadro 3 se presenta la posición de los países en producción científica por cada base de datos.

\begin{tabular}{|c|c|c|c|c|c|c|}
\hline Posición & $\begin{array}{c}\text { Colección } \\
\text { principal } \\
\text { de Web of } \\
\text { Science }\end{array}$ & $\begin{array}{c}\text { Current } \\
\text { Contents } \\
\text { Connect }\end{array}$ & MEDLINE & $\begin{array}{c}\text { SciELO } \\
\text { Citation } \\
\text { Index }\end{array}$ & $\begin{array}{c}\text { KCI - } \\
\text { Korean } \\
\text { Journal } \\
\text { Database }\end{array}$ & $\begin{array}{c}\text { Russian } \\
\text { Science } \\
\text { Citation } \\
\text { Index }\end{array}$ \\
\hline 1 & Brasil & Brasil & Brasil & Brasil & Brasil & México \\
\hline 2 & México & México & México & Colombia & México & Brasil \\
\hline 3 & Argentina & Argentina & Argentina & México & Argentina & Colombia \\
\hline 4 & Chile & Chile & Chile & Chile & Chile & Argentina \\
\hline 5 & Colombia & Colombia & Colombia & Argentina & Colombia & Cuba \\
\hline 6 & Ecuador & Perú & Perú & Cuba & Ecuador & Chile \\
\hline 7 & Perú & Ecuador & Ecuador & Perú & Venezuela & Venezuela \\
\hline 8 & Cuba & Uruguay & Uruguay & Ecuador & Perú & Uruguay \\
\hline 9 & Uruguay & Puerto Rico & Puerto Rico & Costa Rica & Paraguay & Paraguay \\
\hline 10 & Venezuela & Venezuela & Venezuela & Venezuela & Panamá & Puerto Rico \\
\hline 11 & Puerto Rico & Cuba & Cuba & Uruguay & Cuba & Ecuador \\
\hline 12 & Costa Rica & Costa Rica & Panamá & Bolivia & Uruguay & Costa Rica \\
\hline 13 & Panamá & Panamá & Costa Rica & Paraguay & Puerto Rico & Honduras \\
\hline 14 & Paraguay & Bolivia & Paraguay & Panamá & Costa Rica & El Salvador \\
\hline 15 & Bolivia & Paraguay & Guatemala & Puerto Rico & Guatemala & $\begin{array}{l}\text { República } \\
\text { Dominicana }\end{array}$ \\
\hline 16 & Guatemala & Guatemala & Bolivia & Guatemala & Bolivia & Nicaragua \\
\hline 17 & Nicaragua & Nicaragua & $\begin{array}{l}\text { República } \\
\text { Dominicana }\end{array}$ & Honduras & Honduras & Perú \\
\hline 18 & Honduras & Honduras & Nicaragua & El Salvador & El Salvador & Panamá \\
\hline 19 & El Salvador & El Salvador & Honduras & $\begin{array}{l}\text { República } \\
\text { Dominicana }\end{array}$ & $\begin{array}{l}\text { República } \\
\text { Dominicana }\end{array}$ & Guatemala \\
\hline 20 & $\begin{array}{c}\text { República } \\
\text { Dominicana }\end{array}$ & $\begin{array}{c}\text { República } \\
\text { Dominicana }\end{array}$ & El Salvador & Nicaragua & Nicaragua & Bolivia \\
\hline
\end{tabular}

Cuadro 3. Producción científica de los países de Latinoamérica en diferentes bases de datos durante el período 2015 - 2020. Fuente: (Clarivate, 2020, Sección Búsqueda avanzada).

De acuerdo con los datos obtenidos en la plataforma Web of Science, Brasil es el país en Latinoamérica que tiene un mayor número de registros en la Colección Principal de Web of Science (387464), Current Contents Connect (191285), MEDLINE (161923), SciELO Citation Index (90537) y Korean Journal Database (664), por lo cual, se posiciona en el puesto 1. Mientras tanto México tiene más registros en Russian Science Citation Index (85).

Ecuador, con respecto a los otros 19 países latinoamericanos está en la posición 6 en los registros de la Colección Principal de Web of Science, en la posición 7 en Current Contents

Licencia Creative Commons Atribución 4.0 Internacional (CC BY 4.0) 
Connect, en la posición 7 en MEDLINE, en la posición 8 en SciELO Citation Index, en la posición 6 en Korean Journal Database y en la posición 11 junto a Costa Rica, Nicaragua, Honduras, El Salvador y República Dominicana en Russian Science Citation Index.

\section{Discusión de resultados}

Con los datos obtenidos en los resultados, es notable la diferencia que existe entre Brasil, México, Argentina, Chile y Colombia con respecto a Ecuador. A pesar de que los registros obtenidos sitúan a Ecuador en el puesto 7 de la una lista de 20 países en el período 2015 2020, esta comparación solo ha sido hecha a nivel latinoamericano y no europeo.

En relación con las bases de datos de las que se recopiló los resultados sobresale Brasil casi en la totalidad de ellas. Sin embargo, es notable que en Korean Journal Database (KCI) y en Russian Science Citation Index, los países latinoamericanos muestren una cantidad baja de registros. Puede ser provocado por la escasez de alianzas o colaboraciones con grupos investigadores científicos de estos países o investigadores que manejen estos idiomas.

Durante el período 2015 - 2020, el Ecuador se sitúa en la posición 6, 7 u 8 en las diferentes bases de datos obtenidas en la plataforma Web of Science. Esto indica que la producción académica está presente en las diferentes bases de datos, por lo cual, las publicaciones científicas o académicas ecuatorianas están siendo difundidas o utilizadas en otras investigaciones a nivel mundial y forman parte de bases de datos internacionales.

Finalmente, la comparación realizada sobre la producción científica durante los períodos 2006 - 2008 y 2015 - 2020, señalan una mejoría considerable de Ecuador porque pasa de una posición 12 a una posición 7. Además, la tasa de crecimiento del año 2006 con respecto al año 2019 es del 907,44 \%. Puede ser la respuesta a la reforma sobre la acreditación de las Instituciones de Educación Superior en el 2008 y por el impacto de las Tecnologías de la Información y Comunicación (TIC) en todas las áreas académicas y de investigación.

\section{Conclusiones}

La producción académica se produce en su mayoría en las instituciones de educación superior de los países ya que representa un compromiso con la sociedad. De esta forma, la investigación y la difusión de los resultados contribuyen con el desarrollo social y económico de los países.

Pese a los conflictos de carácter económico, social y político que experimentan los países de América Latina, el índice de producción científica en los últimos años ha incrementado considerablemente debido a los cambios o reformas gubernamentales de cada nación y a la intervención de instituciones que se centran en el desarrollo académico.

Actualmente, en las IES del país, la producción científica y su importancia ha sido más priorizada. Sin embargo, existe carencia de conocimiento sobre la producción y redacción de textos académicos por parte de los estudiantes. Esto se da por el paradigma educativo ecuatoriano en el que la escritura no es vista como un eje transversal desde los primeros años de educación formal, estos problemas se van acumulando y finalmente, los resultados en la educación superior no son óptimos.

Ecuador se encuentra en el puesto 7 de los 20 países de Latinoamérica de acuerdo con los datos extraídos de la plataforma Web of Science. En Ecuador, es escasa la importancia que se le da a la producción científica. Por otro lado, este trabajo investigativo solo ha sido

Licencia Creative Commons Atribución 4.0 Internacional (CC BY 4.0)

Revista Cátedra, 3(2), pp. 150-165, mayo-agosto 2020. e-ISSN: 2631-2875

https://doi.org/10.29166/10.29166/catedra.v3i2.2160 
realizado mediante la comparación de los registros a nivel latinoamericano y no europeo o mundial. Los países considerados primermundistas presentan registros más altos en la producción científica y sería importante realizar otro estudio sobre ese tema para analizar la situación de Ecuador a una escala global.

Ecuador ha mejorado significativamente en cuanto a la producción científica a nivel de Latinoamérica del año 2006 a la actualidad. A partir del 2008, se instauró un nuevo mandato con respecto a la acreditación de las Instituciones de Educación Superior. "Las publicaciones científicas de las y los docentes de la IES y que, en principio, se supone presentan los avances y alcances de sus actividades investigativas" (CEAACES, 2014, p. 143). Entonces, las publicaciones científicas de los docentes fueron establecidas como un factor fundamental para determinar la calidad de la educación. Según los resultados, estos lineamientos han influido positivamente en el desarrollo científico y académico en el país ya que se ha dado importancia a la investigación de todas las áreas del conocimiento y a su difusión.

\section{Agradecimientos}

Los autores expresan el agradecimiento a la Magíster Verónica Simbaña-Gallardo por su guía durante el proceso de producción de este artículo.

\section{Bibliografía}

Álvarez Muñoz, P., y Pérez-Montoro Gutiérrez, M. (2016). Análisis de la producción y de la visibilidad científica de Ecuador en el contexto andino (2000-2013). El Profesional de la Información, 24(5), 577-586. doi:10.3145/epi.2015.sep.07

Cañedo Andalia, R., Pérez Machín, M., Guzmán Sánchez, M., y Rodríguez Labrada, R. (2010). Aproximaciones a la visibilidad de la ciencia y la producción científica de Cuba en el sector de la salud. Acimed, 21(1), 28 - 43.

Castillo, J. A., y Powell, M. A. (2019). Análisis de la producción científica del Ecuador e impacto de la colaboración internacional en el período 2006-2015. Revista Española de Documentación Científica, 42(1), 225. doi: 10.3989/redc.2019.1.1567

CEEACES. (2014). La evaluación de la calidad de la universidad ecuatoriana. La experiencia del Mandato 14. Quito, Ecuador: Consejo de Evaluación, Acreditación y Aseguramiento de la Calidad de la Educación Superior. Recuperado el 2 de enero de $2020 \mathrm{de}$ https://issuu.com/ceaacesec/docs/ceaaces 2014 la evaluacio $\mathrm{n}$ de la

Cedeño, C. G. (2014). El proceso extensionista universitario como vía para la pertinencia en la formación del futuro profesional. Revista ESPAMCIENCIA, 5(1), 17-23.

Constitución de la República del Ecuador (2008). [en línea]. Ecuador: Publicación oficial de la Asamblea Constituyente. Recuperado el 15 de enero de 2020 de: https://www.asambleanacional.gob.ec/sites/default/files/documents/old/constit ucion de bolsillo.pdf

Clarivate, 2019. Colección principal de Web of Science Ayuda. Web of Science. Recuperado el 22 de enero de 2020 de https://images.webofknowledge.com//WOKRS534DR1/help/es LA/WOS/hp dat abase.html.Ecuador . Asamblea Constituyente. (2008). Constitución de la República. Montecristi, Ecuador. 
Clarivate, 2020. Web of Science. Recuperado el 8 de enero de 2020 de: https://apps.webofknowledge.com/UA AdvancedSearch input.do?SID=6AiJET6gS $\underline{\mathrm{dD} 4 h S B X j N f \& p r o d u c t=U A \& \text { search mode=AdvancedSearch }}$

FECYT. (2019). Bases de Datos de Web of Science. Recursos científicos. Recuperado el 19 de diciembre de: https://www.recursoscientificos.fecyt.es/licencias/productoscontratados/wos

Guerrero-Casado, J. (2017). Producción científica latinoamericana indexada en Scopus en el área de las ciencias agropecuarias: análisis del período 1996-2016. Idesia (Arica), 35(4), 27-33.

Ley Orgánica de Educación Superior. Registro Oficial N 77 de 15 -may-2000. QuitoEcuador, 12 de oct. de 2010. Recuperado el 16 de enero, 2020 de: http://www.ces.gob.ec/index.php?option=com phocadownload\&view=category\&i $\underline{\mathrm{d}=11 \text { :ley-organica-de-educacion-superior\&Itemid }=137}$

Piedra-Salomón, Y., y Martínez-Rodríguez, A. (2007). Producción científica. Ciencias de la Información, 38(3), 33-38.

Rojas, J. E. (2011). Reforma universitaria en el Ecuador. Etapa de transición. Innovación Educativa, 11(57), 59-67.

Russell, J., Ainsworth, S., Del Río, J., Narváez-Berthelemot, N., y Cortés, H. (2007). Colaboración científica entre países de la región latinoamericana. Revista Española de Documentación Científica, 30(2), 180 - 198. doi: 10.3989/redc.2007.v30.i2.378

Santa, S., y Herrero Solana, V. (2010a). Cobertura de la ciencia de América Latina y el Caribe en Scopus vs Web of Science. Investigación bibliotecológica, 24(52), 13-27.

Santa, S., y Herrero Solana, V. (2010b). Producción científica de América Latina y el Caribe: una aproximación a través de los datos de Scopus (1996-2007). Revista Interamericana de Bibliotecología, 33(2), 379-400.

UAM Biblioteca (2019). Web of Science (WOS): Inicio. UAM_Biblioteca. Recuperado el 22 de enero de 2020 de: https://biblioguias.uam.es/tutoriales/WOS

UNESCO (2015). Informe de la UNESCO sobre ciencia, hacia el 2030. París, Francia. $\begin{array}{llllll}\text { Recuperado el } & 15 \text { de } & \text { enero de }\end{array}$ http://unesdoc.unesco.org/images/0023/002354/235407s.pdf 


\section{Autores}

ELKING ARAUJO-BILMONTEobtuvo su título de Magíster en Gerencia y Liderazgo educacional por la Universidad Técnica Particular de Loja en 2014. Obtuvo su título de Máster en Lexicografía Hispánica por la Asociación de Academias Americanas de la Lengua en 2002. Obtuvo su título de Licenciado en Ciencias de la educación, especialización Lengua y Literatura por la Universidad Central del Ecuador en 1998.

Actualmente es docente investigador de la Facultad de Comunicación, Lingüística y Literatura de la Pontificia Universidad Católica del Ecuador.

LICETH HUERTAS-TULCANAZA obtuvo su Certificado de Proficiencia en Inglés en el Centro de Educación Continua de la Escuela Politécnica Nacional en Quito (Ecuador) en 2019.

Actualmente es estudiante de sexto semestre de la Carrera de Pedagogía de la Lengua y la Literatura, de la Universidad Central del Ecuador.

KENNY PÁRRAGA-STEAD actualmente es estudiante de sexto semestre de la Carrera de Pedagogía de la Lengua y la Literatura, de la Universidad Central del Ecuador. 